\title{
Christianisme, interprétation et écriture
}

Quelques réflexions sur l'histoire occidentale des rêves

Christianity, Interpretation, and Writing. Reflections on the Western History of Dreams

Jacqueline Carroy

\section{OpenEdition} Journals

\section{Édition électronique}

URL : https://journals.openedition.org/extremeorient/1201

DOI : 10.4000/extremeorient.1201

ISBN : 978-2-84292-990-9

ISSN : 2108-7105

Éditeur

Presses universitaires de Vincennes

\section{Édition imprimée}

Date de publication : 1 décembre 2018

Pagination : 239-252

ISBN : 978-2-84292-988-6

ISSN : 0754-5010

Référence électronique

Jacqueline Carroy, "Christianisme, interprétation et écriture », Extrême-Orient Extrême-Occident [En ligne], 42 | 2018, mis en ligne le 01 décembre 2020, consulté le 08 janvier 2022. URL : http:// journals.openedition.org/extremeorient/1201 ; DOI : https://doi.org/10.4000/extremeorient.1201 


\title{
Christianisme, interprétation et écriture Quelques réflexions sur l'histoire occidentale des rêves
}

\author{
Jacqueline Carroy
}

Confrontée au défi de poser un regard extérieur de non-spécialiste à propos du monde des rêves que j'ai découvert dans cette publication, je souhaiterais proposer quelques éléments de réflexion inspirés par mes propres recherches et par des lectures concernant surtout l'Occident. Bien entendu, je ne prétends évoquer que quelques discordances et concordances, sans souci d'exhaustivité, entre ces textes sur la Chine, la Corée et le Japon et ce que je peux avancer sur un ailleurs occidental. La perspective que j'adopterai, dans la lignée de plusieurs travaux littéraires, anthropologiques et historiques, dont on pourra lire les références en bibliographie, visera à souligner des spécificités historiques plutôt qu'un « de tout temps » ou un « en tous lieux ».

Voir et entendre en dormant ou en somnolant n'en est pas moins un fait très général, voire universel, sans doute commun aux humains et aux animaux, comme le remarquait Aristote. Mais le rêve n'est pas un simple phénomène animal. Il a d'entrée de jeu une dimension culturelle et sociale au sens large : il est un support de croyance, de récit, de conte, de poésie, de divination, de thérapie, etc. Il fait l'objet de mises en science et en laboratoire en Occident depuis le XIX ${ }^{e}$ siècle. Ce dernier point relève du constat plus que du jugement de valeur et ne veut pas dire que le cerveau rêvant donne le dernier mot des phénomènes oniriques, si tant est qu'il y ait un dernier mot. En d'autres termes, il me semble important de s'abstenir autant que possible de concevoir l'histoire des rêves en termes de progrès ou d'évolution allant inéluctablement vers une conception qui serait forcément plus vraie parce que du ressort d'une psychanalyse, voire d'une sociologie, s'affichant universelles, ou encore d'une neurobiologie aux prestiges désormais grandissants.

Cela ne veut pas dire que les savoirs et les pratiques onirologiques soient incommensurables et incomparables. À la lecture de cette revue, on peut repérer ainsi, entre Orient et Occident, des situations et des expériences communes - s'assoupir, ne pas savoir si l'on veille ou dort, se réveiller pour 
se dire « ce n'était qu'un rêve », voir des morts ou avoir des visions érotiques, voir l'avenir de façon obscure ou claire, inventer en dormant, faire littérature des rêves. Autant de vécus qui peuvent être, ici et là, vecteurs de croyances, de métaphores, d'intrigues romanesques ou théâtrales, de politiques, d'invites à création poétique ou théorique. Beaucoup des usages des rêves mis en exergue dans les articles de ce numéro pourraient avoir des analogies à l'Ouest : ce point me frappe par rapport au corpus des exemples savants à partir duquel j'ai tenté pour ma part d'écrire une histoire des rêves au XIX ${ }^{\mathrm{e}}$ siècle. C'est pourquoi des songes étrangers peuvent intéresser et impliquer une lectrice contemporaine. La lecture des articles de ce numéro m'a donné envie de décentrer mon point de vue sur l'histoire de l'onirologie occidentale et de voir celle-ci comme si je me situais ailleurs, plus précisément dans un entre-deux ou dans un va-et-vient qui ferait apparaître en retour « mon » monde des rêves comme proche, étrange et singulier, tout à la fois.

C'est ce type d'exercice que je souhaiterais esquisser en tendant une sorte de miroir comparatif et en proposant quelques réflexions que pourra compléter la bibliographie jointe à cet article. Je proposerai un portrait du rêveur occidental en rêveur chrétien. Je m'interrogerai sur des rapports, obsédants mais jamais tout à fait légitimes, avec l'interprétation de l'avenir et la divination. J'essaierai enfin pour conclure d'avancer une comparaison possible qui me semble importante et qui tournerait autour de l'écriture des rêves.

Peut-être faut-il commencer par quelques brèves remarques préliminaires de vocabulaire. Lorsque les clercs du Moyen Âge écrivent des récits de rêve, ils emploient les termes latins de somnium ou de visio. À partir du XVII ${ }^{\mathrm{e}}$ siècle, « rêver », renvoyant à l'époque médiévale au fait de divaguer, d'aller de-ci de-là et d'extravaguer entre en concurrence avec « songer » et prend son sens actuel, tandis que « rêve » et « rêveur » peuvent désigner toujours une errance ou une personne de tempérament mélancolique. Tous ces mots en viennent à renvoyer, au XIX ${ }^{\mathrm{e}}$ siècle, au même type de phénomène nocturne et parfois diurne, comme en témoignent les dictionnaires. Alors que «songer » et « songeur » ne sont plus directement liés au sommeil, « songe »n'est cependant pas supplanté et prend actuellement un sens plus poétique ou plus recherché. Contrairement à d'autres langues européennes comme l'anglais, l'allemand, l'italien ou l'espagnol, le français a donc aujourd'hui la particularité de disposer de deux vocables, « rêve » (un « mot sans pédigrée », pour reprendre Daniel Fabre) et « songe » (qui dérive sans surprise du latin somnium) ${ }^{1}$.

1. Voir Besson et Schmitt $2017: 13-15$; Fabre $1996: 69-82$. 


\section{Le christianisme et la psychologisation des rêves}

Un premier constat massif, en forme de truisme. La lecture de ce numéro m'a amenée à comprendre l'importance de l'encadrement des rêves par le confucianisme, le taoïsme et le boudhisme. Il y aurait lieu, probablement, comme je m'en suis aperçue, d'affiner et de mettre au pluriel ces termes de diverses manières. De même, dans le contexte chrétien que je mettrai en avant, il faudrait spécifier de quel christianisme on parle. Dans le cadre limité de cet essai, je m'en tiendrai au christianisme occidental ${ }^{2}$.

Les travaux pionniers de Jacques Le Goff remettent en cause les périodisations habituelles et avancent le thème d'un «long Moyen Âge » occidental, notion féconde si l'on veut écrire une histoire des rêves. L'historien montre bien que le christianisme a encadré et encadre les songes occidentaux, en induisant une méfiance durable à leur égard ${ }^{3}$. Les visions et voix nocturnes sont signes d'aliénation, au sens étymologique, du sujet chrétien, soumis à ces « autres » que sont Dieu, les anges et le diable, mais aussi marqué par une nature peccamineuse. Le christianisme naissant reprend en la dramatisant et en l'assombrissant l'onirologie antique. Sans entrer dans le détail de classifications qui ont été reprises pendant des siècles, celle-ci distinguait presque toujours le rêve vrai qui dévoile et a un sens par rapport à l'avenir (appelé souvent oneiros) et le rêve naturel (désigné souvent sous le vocable d'ennupnion) insignifiant ou trompeur, porteur de besoins du corps ou de préoccupations de la veille 4 . Dans un passage célèbre d'Homère toujours cité et repris notamment en 1855 par Gérard de Nerval dans l'incipit d'Aurelia, le premier type de vision passait par des portes de corne et le second par des portes d'ivoire.

C'est dans cet héritage antique, mais aussi en rupture avec celui-ci, que se développe au Moyen Âge un genre littéraire de l'autobiographie et que sont transcrits des « rêves de soi » qui sont la plupart du temps des rêves de clercs : le statut d'autorité des prêtres et des moines autorise la divulgation de ce type de songes à titre d'exemples. Ceux que donnent à lire Gisèle Besson et Jean-Claude Schmitt dans leur passionnante anthologie témoignent, pour beaucoup, des tourments de rêveurs qui s'interrogent sur des visions nocturnes ou diurnes dont l'origine leur est incertaine : sont-elles divines, diaboliques ou simplement venues du corps et de l'esprit du dormeur ? Ces songes témoignent d'une interrogation récurrente sur la sexualité et la culpabilité sexuelle : «Il ne fait pas de doute que les rêves médiévaux, qui mettent souvent en scène les

2. Sur les rêves byzantins, voir par exemple Timotin 2010 .

3. Le Goff $1977,1985$.

4. Pour des analyses plus précises, voir Chandezon 2018 : 77-116. 
tentations du démon, sont aussi l'expression d'une forte culpabilité morale, que l'Église s'efforce, surtout à partir du XIII ${ }^{e}$ siècle, d'orienter vers l'aveu, la confession des péchés dans le cadre du sacrement de pénitence ${ }^{5}$. » Il faut, évidemment, souligner, rappellent Besson et Schmitt, que nous ne savons rien, ou pas grand-chose, des rêves non écrits de ceux qui ne sont ni clercs, ni nobles, ni rois, ni mâles.

Ces auteurs évoquent une «neutralisation » au long cours de l'onirologie chrétienne qui aboutirait à sa psychologisation à partir des XVII ${ }^{\mathrm{e}}$ et XVIII ${ }^{\mathrm{e}}$ siècles et s'achèverait, pourrais-je ajouter, aux $\mathrm{XIX}^{\mathrm{e}}$ et $\mathrm{XX}^{\mathrm{e}}$ siècles : " L'enjeu était donc que fut reconnue en l'homme une faculté imaginative autonome, où les rêves pussent naître à l'abri des influences extérieures, devenir neutres sur le plan moral et apparaître pleinement comme étant le "propre de l'homme". La théologie scolastique, la médecine de la fin du Moyen Âge, la philosophie de l'époque moderne ont forgé tour à tour les catégories qui ont permis de dégager ce champ immense de la psychologie humaine ${ }^{6}$. » Et de fait, lorsqu' on consulte, à l'autre bout de l'histoire, l'article "Songe» du Dictionnaire de cas de conscience de Jean Pontas, les références à Dieu et au Diable, sans disparaitre, se sont plus ou moins estompées et il est affirmé que « la plupart des songes sont autant d'idées creuses et vaines ${ }^{7} \gg$.

C'est au XIX ${ }^{\mathrm{e}}$ siècle que prédomine et s'impose, dans le monde savant et cultivé des philosophes, des médecins et des amateurs de rêves, l'idée qu'il ne faut plus classifier les songes en vrais et faux et qu'ils renvoient tous aux sensations internes du corps (souvent des organes génitaux) et du cerveau, transforment des perceptions extérieures en les exagérant, procèdent d'associations d'idées, et renvoient à l'imagination ou à la mémoire du rêveur. Dans cette approche, dont l'historien Alfred Maury et le marquis et sinologue Léon d'Hervey de Saint-Denis sont des représentants emblématiques ${ }^{8}$, les rêves ne renvoient surtout pas à l'avenir, mais procèdent de facultés comme la mémoire au premier chef. Il ne s'agit alors pas d'interpréter, ce qui pourrait renvoyer à un retour aux «superstitions », mais d'analyser des mécanismes de formation des visions et des voix nocturnes.

\footnotetext{
5. Besson et Schmitt $2017: 12$.

6. Ibid.: 42 .

7. Pontas 1847 : 665-666. Le dictionnaire reprend et adapte une édition du XVIII ${ }^{\mathrm{e}}$ siècle.

8. Maury 1861 ; Hervey de Saint-Denys 1867. Sur Maury, voir Carroy et Richard 2007. Bien qu'il ait noté et dessiné assidument ses propres rêves, Hervey de Saint-Denys ne semble pas s'être intéressé aux rêves chinois. Sur sa carrière de sinologue, voir Pino 1995 : 95-129.
} 
Et, pour ce faire, il faut produire et publier des nouveaux récits oniriques fiables, généralement personnels, qui remplacent les recueils d'anecdotes merveilleuses héritées de l'Antiquité et de la Bible. Se construit ainsi un nouveau répertoire d'exemples censés être scientifiques qui deviennent classiques au cours du siècle. Un des rêve de Maury, illustrant la rapidité de l'esprit endormi, publié en 1853 puis en $1861^{9}$ - dans lequel le dormeur se voit jugé et condamné à la guillotine sous la Terreur, pour se réveiller ensuite en sursaut, la flèche au-dessus de son lit étant tombée sur son cou - suscite de multiples commentaires, y compris dans la presse. "Maury guillotiné » est cité et présenté dans le journal Le Gaulois du 11 décembre 1877 comme une illustration de l'imagination sans limite du dormeur. Le rêve suscite une relecture de Freud. Lorsque ce dernier deviendra une figure dominante de l'onirologie occidentale, c'est le rêve dit de l'injection faite à Irma, mis en exergue et en modèle en ouverture de L'Interprétation $d u$ rêve ${ }^{10}$, qui fera figure de « rêve des rêves », pour reprendre une formule de Lacan.

La caractéristique des exemples savants juste antérieurs à Freud (ou contemporains) est d'être souvent en première personne et de convoquer un « intime scientifique » et parfois autobiographique. À côté de rêves professionnels situés à la Bibliothèque de l'Institut, dont il est membre, Maury se met aussi en scène dans des épisodes nocturnes où il voit et entend des morts dont le deuil a été douloureux ou impossible. Son père catholique (décédé en 1831 d'une tumeur au cerveau) est associé à une guérison miraculeuse par le sang d'un saint, ou encore son frère cadet « tant regretté » qui s'est suicidé en 1849 réapparaît vivant dans un rêve daté de 1861. Maury révèle avec franchise la cause de ces morts dans des Souvenirs manuscrits dictés après 1870 et déposés à l'Institut pour être divulgués de façon posthume. Les passages concernant notamment le père et le frère témoignent d'une volonté d'aveu ou de confession par rapport aux exemples oniriques imprimés antérieurement. Si Maury a perdu la foi de son enfance et s'il est voltairien ou déiste le jour, il reste la nuit hanté par les « superstitions » catholiques. Le contenu de certains de ses rêves renverrait à un long Moyen Âge chrétien dont on ne peut se défaire et dont témoigne le titre à rallonge d'un livre, La Magie et l'astrologie dans l'Antiquité et au Moyen Âge ou étude sur les superstitions païennes qui se sont perpétuées jusqu'à nos jours ${ }^{11}$. Les superstitions n'en finissent pas, notamment parce que nous dormons et rêvons. Dans le rêve de 1861 où revit fugitivement le frère

9. Maury $1861: 133-134$.

10. Freud $2010: 143-159$.

11. Maury 1860 . 
«tant regretté », Maury évoque le « véritable plaisir » qu'il a eu à son réveil ${ }^{12}$. Tout se passe comme si ce moment de joie fugace palliait une croyance ferme dans les revenants devenue impossible. Si Maury emploie parfois le terme de fantôme pour parler du rêve et de ses rêves, c'est en se conformant à l'usage de la psychologie de son temps notamment à Maine de Biran, en pensant au sens du mot pour le bibliothécaire et bibliophile qu'il est (la marque d'un livre manquant sur une étagère) et sans doute aussi en se référant à Hamlet. Shakespeare autorise une croyance littérarisée dans le retour des morts. Si l'on se réfère à l'anthologie de rêves de la famille Yu constituée à partir du début du XX $X^{\mathrm{e}}$ siècle et étudiée par Rania Huntington, une hésitation analogue subsiste quant au statut des défunts vus la nuit : souvenirs ou revenant ${ }^{13}$ ? À chaque société ses fantômes, « laïques » ou non, et ses cultes des morts.

Désormais le réveil neutraliserait et psychologiserait le contenu de ce que l'on a rêvé. Pour le dire autrement, il y aurait seulement des rêves banals qui passeraient exclusivement par des portes d'ivoire. Telle pourrait être la marque d'une modernité occidentale qui procèderait du christianisme sur une très longue durée. Mais ce récit est trop linéaire et cette modernité, on va le voir, est relative. Pour ne donner qu'un exemple, au moment où Maury rêve, l'apparition nocturne de son père et de son frère auraient pu le faire pencher vers une toute nouvelle foi relevant du spiritisme, qui se répand aux États-Unis puis en Europe au cours du XIX siècle $^{14}$. Au moment même où les rêves se sécularisent, les spectres et la divination se modernisent. Il faut donc proposer un fil historique plus sinueux ou plus complexe.

\section{Interpréter par rapport à l'avenir ou au passé ?}

Une tradition perdure et coexiste depuis l'Antiquité et le Moyen Âge, qui assigne aux songes un rôle prémonitoire. L'interdit est jeté par la Bible, puis par l'Église dès ses débuts, de façon réitérée, sur la divination et la magie. De ce fait, une rupture aurait pu s'établir entre d'une part des visions de l'Ancien et du Nouveau Testament, et d'autre part les songes banals de tout un chacun dont le caractère prédictif aurait été voué à condamnation et destiné à disparaître. L'imitation de Jésus ne peut jouer pour justifier ou « sauver » ce type de rêve : au mont des Oliviers, ce sont les disciple qui dorment et Jésus qui veille et doute. Contrairement à la tradition concernant Confucius et aux récits

12. Maury $1861: 96$.

13. Huntington 2017.

14. Edelman 1995 ; Cuchet 2012. 
du Coran et des hadîths sur Mahomet (qui fut un grand rêveur) ${ }^{15}$, les Évangiles ne rapportent aucun songe du Christ. Celui-ci n'aurait pas rêvé, à la manière d'un sage chinois célèbre ${ }^{16}$, si l'on peut risquer cette analogie.

Une comparaison avec la société musulmane du Moyen Âge est éclairante concernant l'interprétation des rêves. L'islam choisit en quelque sorte de « laïciser » la divination et accueille l'existence de devins qui n'ont pas une fonction religieuse : "On ne considérait plus la connaissance divinatoire comme le fruit d'une inspiration divine, mais comme l'acquis d'une science humaine ${ }^{17}$. » À l'inverse, l'Église chrétienne condamne avec insistance une demande de divination venue de la société. Mais elle tolère certaines pratiques comme les sorts bibliques (ouvrir au hasard une page des Évangiles pour y voir l'avenir). Surtout, elle valorise et encourage une hagiographie qui multiplie les récits de rêves prémonitoires attribués aux saints (rêves de leurs mères avant leur naissance, rêves de vocation, etc. ${ }^{18}$. Désormais seuls les candidats à la canonisation sont censés pouvoir avoir des rêves vrais, contrairement à ce qu'affirmait Aristote qui imputait plutôt ceux-ci aux hommes ignorants et sots. Sur un autre plan, une connaissance scientifique de l'avenir est reconnue par ailleurs à l'astrologie, qui ne se sépare pas, jusqu'au XVII ${ }^{\mathrm{e}}$ siècle, de l'astronomie et que princes, papes et hauts personnages considèrent comme un savoir légitime. Celle-ci renaîtra de ses cendres au XIX ${ }^{\mathrm{e}}$ siècle, puis prospérera sous une forme plus ou moins ludique dans nos journaux contemporains ${ }^{19}$.

Les inquiétudes sur l'avenir et le besoin de prédire n'en demeurent pas moins vivaces. Il faut ici évoquer l'abondante production onirocritique qui s'adresse aux lecteurs occidentaux, du Moyen Âge à nos jours. On passe, dans cette littérature, de la première à la troisième personne du singulier des récits de rêves de soi ou de rêves d'autrui, à une deuxième personne qui parle souvent à un lecteur rêveur (éventuellement acheteur) en disant «tu » ou « vous ». Ce lecteur, déjà quelque peu cultivé puisqu'il sait lire, consulte des listes alphabétiques manuscrites de rêves potentiels au Moyen Âge, puis des dictionnaires imprimés assignant à chaque vision nocturne un ou parfois des sens circonscrits. Les listes du Moyen Âge sont christianisées et attribuées souvent au prophète biblique Daniel. À partir du XVI siècle, on redécouvre Artémidore, l'auteur ancien de la seule onirocritique antique conservée

15. Lory $2003: 30 s q$.

16. Diény 2001.

17. Fahd cité par Boglioni $2000: 45$.

18. Boglioni $2000: 51-60$.

19. Edelman 2006. 
presque en entier ${ }^{20}$. Il est édité, traduit, imprimé et diffusé en grand nombre, parfois passé au tamis des onirocritiques arabes, parfois censuré, dans toute l'aire occidentale.

Les clés des songes, comme on les appellera en français au XIX ${ }^{\mathrm{e}}$ siècle, ont un statut paradoxal de publications à la fois courantes et marginales de diverses manières. Alfred Maury rapporte que, dans les rues de Paris, on vendait des clés des songes aux cris de : «Avez-vous rêvés de chien ? Avezvous rêvé de chat $^{21}$ ? ». Diffusées par colportage ou dans des magasins équivalant à des bazars, ces clés des songes prennent parfois l'allure de brochures sommairement imprimées et éditées dans des collections dites populaires, mais aussi plus soignées, correspondant à un public bourgeois et mondain. Ces publications s'adressent de plus en plus aux femmes et portent parfois la signature de voyantes professionnelles qui pratiquent en parallèle différents types de divination, par l'astrologie, les cartes, le marc de café, le somnambulisme provoqué (qu'on appelle aussi l'hypnotisme), etc. Les clés des songes acquièrent un usage ludique, au sens propre et figuré. Elles prêtent à demi croyances et à plaisanteries parfois grivoises. La remémoration des rêves est aussi, à partir du XVIII ${ }^{\mathrm{e}}$ siècle (au moment où s'instaurent en Europe des loteries plus ou moins interdites ou organisées par les états), supposée permettre de jouer au loto, chaque vision onirique étant associée à un chiffre ou un nombre à jouer. Cet usage se prolonge actuellement à Naples, où l'on peut acheter une Smorfia (littéralement « grimace »), un dictionnaire pour miser ses rêves. Au moment où les angoisses s'aiguisent, sous la Grande Guerre, se vend en France une clé des songes modernisée intégrant l'argot des tranchées dans son répertoire. Elle s'adresse prioritairement aux femmes qui rêvent à l'arrière, comme le montre sa couverture, mais elle est de taille suffisamment réduite pour se glisser dans la poche d'un uniforme militaire. Il est difficile de savoir si cette brochure a eu du succès et si elle n'a constitué qu'une exception. Actuellement les clés des songes se publient sur internet, parées parfois du prestige d'une interprétation renouvelée par des références à Freud ou Jung.

Les prophéties n'ont pas non plus disparu au cours de l'histoire occidentale. On pourrait illustrer ce point à partir de l'exemple assez significatif d'une publication médicale emblématique à plus d'un titre. Les Annales médico-psychologiques, fondées en 1843, sont la revue professionnelle des aliénistes, ceux qu'on appellera plus tard les psychiatres, qui ont en charge l'administration des asiles et les soins des aliénés. La société du même nom

20. Chandezon et du Bouchet 2014. Pour une histoire occidentale des clés des songes, voir Carroy et Lancel 2016.

21. Maury $1862: 589$. 
rassemble non seulement une majorité de médecins mais aussi des philosophes et des non-médecins (comme Maury). Parmi les médecins liés à la revue et à la société qui se définissent comme des spiritualistes, Maurice Macario, exilé piémontais, travaille à Bicêtre. Après avoir publié sur les hallucinations, un mot qui se répand alors en France dans le domaine médical et littéraire, il aborde les rêves dans deux articles de 1846 et 1847 . Reprenant sous d'autres noms des classifications anciennes, il met en exergue, sous le nom de rêves «psychiques » ou intuitifs, des rêves véridiques qu'il oppose aux rêves « intracrâniens » ou "extra-crâniens » comme aux rêves pathologiques qui sont des symptômes ou des prodromes de maladie. Il propose un compendium ou une compilation de toute une tradition héritée de l'Antiquité, du christianisme et de la médecine antique assignant aux rêves un rôle prédictif, aussi bien médical que non médical. À l'appui de l'existence de rêves psychique, il cite de longues prophéties portant sur la Révolution française et des nouvelles fantastiques de Charles Nodier traitées comme des cas médicaux. Les articles de Macario seront réédités dans un livre de 1857 qui deviendra une référence assez importante au XIX ${ }^{\mathrm{e}}$ siècle.

En 1848, toujours dans les Annales médico-psychologiques, Alfred Maury publie un article portant sur ce qu'il nomme « hallucinations hypnagogiques », ces visions qui préludent à l'endormissement et se situent dans un état intermédiaire entre veille et sommeil. À l'anthologie éclectique de Macario, Maury oppose un recueil, censé être directement basé sur des faits d'autoobservation et sur une enquête menée auprès de sa mère, un cousin, une servante. La recherche se fait dans l'entourage proche : on pourrait sur ce point comparer l'anthologie de Maury et celle de la famille Yu décrite par Rania Hutington. Cependant, par-delà les anecdotes familières et familiales, le rêveur français vise un groupe de savants et de médecins susceptible de vérifier ses hypothèses en collectant de nouveaux exemples épurés de merveilleux. Il ne s'agit pas de souder un clan familial par le culte d'ancêtres prestigieux et d'une jeune morte apparus la nuit ${ }^{22}$, mais de créer une communauté de savants rêveurs. Il n'en reste pas moins que les exemples scientifiques publiés par Maury peuvent parfois convoquer en sous-main des revenants laïques, ce qui rapprocherait son anthologie de celle de la famille Yu. De ce point de vue, en dépit de différences évidentes, ces rêveurs et rêveuses chinois qui voient des morts peuvent susciter des échos dans la culture européenne, comme le montre Hutington.

Pour revenir sur la publication presque simultanée dans une même revue médicale, entre 1846 et 1848, d'articles reposant sur l'allégation d'exemples

22. Huntington 2017. 
et d'anecdotes de nature différente, voire opposées, l'histoire des onirologies occidentales pourrait s'écrire, selon un autre fil historique, en termes de stratification et de coexistence autant que de progrès ${ }^{23}$.

La découverte freudienne peut de même apparaître autant comme une rupture que comme la réactivation de mémoires et de traditions. Freud revendique de réhabiliter l'interprétation des rêves populaire héritée d'Artémidore. Cependant, comme ses confrères savants contemporains, il rattache tous les rêves au passé proche ou lointain du dormeur et se garde bien de leur assigner un sens prémonitoire ou prophétique ${ }^{24}$. L'une des grandes innovations de la psychanalyse consiste à ériger une version profondément remaniée de l'onirocritique en pratique thérapeutique. On voit ainsi apparaître en Occident des interprètes professionnels légitimes qui affirment leurs compétences sur tous les rêves humains en fonction non plus d'un avenir mais d'un passé individuel, voire collectif. Ces nouveaux herméneutes que sont les psychanalystes pourraient en effet décrypter non seulement les rêves de leurs analysants situés dans une relation orale à deux décrite en terme de transfert, mais aussi des songes écrits et transmis dans l'histoire et la culture. Freud semble avoir été prudent sur ce dernier point : il hésite à vouloir analyser après coup des rêves attribués à Descartes, en faisant valoir que celui-ci n'a pas été son patient ${ }^{25}$. Mais il se fait fort aussi souvent d'interpréter contes, mythes, folklore, romans et nouvelles comme s'ils étaient des symptômes relevant de plein droit de sa compétence.

\section{Ce que l'écriture fait aux rêves et ce que les rêves font à l'écriture}

Je souhaiterais conclure sur une proposition de comparaison. Contrairement à certaines sociétés traditionnelles dans lesquelles on les raconte et les transmet oralement seulement, en Occident et en Extrême-Orient, nous écrivons nos rêves et publions depuis longtemps des récits oniriques. Plus précisément, nos sociétés mettent en jeu un partage entre, d'une part, ceux qui disent et, d'autre part, ceux qui ne se contentent pas de dire mais consignent ou inventent des rêves écrits, en d'autres termes des lettrés, des clercs, des gens cultivés.

À lire les articles de ce numéro, il m'a semblé que la littérature classique chinoise, japonaise ou coréenne fait beaucoup plus de place aux rêves que la littérature européenne, à de notables exceptions près : pensons au romantisme

23. Dumora-Mabille $2003: 19$.

24. Mayer $2016: 157-181$.

25. Freud $1981: 5$. 
ou au surréalisme par exemple. On ne doit pas oublier cependant que le Moyen Âge européen développe une rhétorique du rêve poétisé enchâssé dans un autre récit, ou encore de l'allégorie onirique (Le Roman de la rose). Le songe est un genre littéraire à l'époque classique : "Le songe d'Athalie » de Racine en est l'exemple le plus connu ${ }^{26}$. Ces songes ont pu devenir des fables et des pamphlets religieux ou politiques ${ }^{27}$. On trouverait trace de cet usage dans un rêve freudien qui fait allusion à Alfred Dreyfus sur l'île du Diable : Freud prend ainsi discrètement parti dans une affaire qui passionne alors toute l'Europe ${ }^{28}$. Au XIX ${ }^{\mathrm{e}}$ siècle, se développe, en dehors du romantisme, un genre du rêve réaliste qui prend partiellement modèle sur l'onirologie contemporaine, comme c'est le cas de Flaubert, un ami de Maury ${ }^{29}$. Et, bien sûr, la psychanalyse et le surréalisme ont inspiré une littérarisation des songes et ont fait se multiplier les rêves écrits ${ }^{30}$. Compte tenu des statuts différent que peuvent avoir le littéraire ou le « lettré » en Occident et en Orient, que veut dire faire littérature des rêves et publier des textes de fiction se référant au rêve?

Dans le domaine des savoirs, le fait d'écrire des rêves pose la question d'une fictionnalisation, et jette un doute sur la véracité des exemples allégués. Le songe bien français de Maury guillotiné - qui semble avoir été très fréquent avec des variantes à l'époque - attise ainsi à la fin du XIX ${ }^{e}$ siècle une polémique sur sa trop grande logique : n'est-ce pas un rêve trop raconté et par là même simplifié et stylisé ? À la suite des psychologues de cette fin de siècle et surtout de Freud, la littérature du $\mathrm{XX}^{\mathrm{e}}$ siècle sera en quête d'une nouvelle écriture onirique qui désarticule éventuellement les codes narratifs habituels pour mieux être au plus près du monde nocturne ${ }^{31}$. Le fait de noter, de transcrire et de publier des rêves aiguise en tous les cas des interrogations sur leur flou et leur labilité, sur la fixation difficile ou impossible des visions et des voix nocturnes, sur l'art de les écrire et de les rendre malgré tout et, plus généralement, sur des frontières indécises entre songe et veille, thèmes que l'on retrouverait, mutatis mutandis, dans plusieurs des articles de cette livraison.

Cette saisie problématique des songes par la plume et le pinceau nous amènerait à nous demander plus spécifiquement, de diverses manières, ce que l'écriture fait aux rêves et ce que les rêves font à l'écriture.

26. Demaules 2010, 2016 ; Dumora 2005.

27. Gantet 2010a, 2010b.

28. Freud $2010: 205$.

29. Vatan 2017 : 50-61.

30. Alexandrian 1974 ; Gollut 1993.

31. Carroy 2012 : 270-283 ; Gollut 1993. 


\section{BIBLIOGRAPHIE}

ALEXANDRIAN Sarane (1974). Le Surréalisme et le rêve. Paris, Gallimard.

BERGÈRE Marie-Claire et PINO Angel (dir.) (1995). Un siècle d'enseignement du chinois à l'École des langues orientales. 1840-1945. Paris, L'Asiathèque.

BESSON Gisèle et SCHMITT Jean-Claude (2017). Rêver de soi. Les songes autobiographiques au Moyen Âge. Toulouse, Anacharsis.

BogLIONI Pierre (2000). «L'Église et la divination au Moyen Âge, ou les avatars d'une pastorale ambiguë ». Théologiques, 8(1) : 37-66.

BonNOT Marie et LEBLOND Aude (dir.) (2017). Les Contours du rêve. Les sciences du rêve en dialogue. Paris, Hermann.

BURKE Peter (1973). «L'histoire sociale des rêves ». Annales, Économies, Sociétés, Civilisations, 28(2) : 329-342.

CARroy Jacqueline (2012). Nuits savantes. Une histoire des rêves (1800-1945). Paris, Éditions de l'EHESS.

CARROY Jacqueline (dir.) (2017). « Rêver au XIX siècle ». Romantisme, 178.

CARroy Jacqueline et LANCEL Juliette (dir.) (2016). Clés des songes et sciences des rêves. De l'Antiquité à Freud. Paris, Les Belles Lettres.

CARROY Jacqueline et RICHARD Nathalie (dir.) (2007). Alfred Maury, érudit et rêveur. Les sciences de l'homme au milieu du XIX siècle. Rennes, PUR.

Chandezon Christophe (2018). " Comprendre et classer les rêves d'Homère à Artémidore ». In DIETERLE Bernard et ENGEL Manfred (dir.) (2018). Theorizing the Dream. Savoirs et théories du rêve. Würzburg, Königshausen \& Neumann : 77-116.

ChANDEZON, Christophe et DU BouCHET, Julien (dir.) (2014). Études sur Artémidore et l'interprétation des rêves. Quatorze études. Paris, Les Belles Lettres.

CUCHET Guillaume (2012). Les Voix d'outre-tombe : tables tournantes, spiritisme et société au XIX $X^{e}$ siècle. Paris, Seuil.

DAUVOIS Nathalie et GROSPERRIN Jean-Philippe (dir.) (2003). Songes et songeurs (XIIIXVIII $e^{e}$ siècles). Laval, Presses de l'Université de Laval.

DÉChanet-Platz Fanny (2008). L'Écrivain, le sommeil et les rêves (1800-1945). Paris, Gallimard.

Demaules Mireille (2010). La Corne et l'Ivoire : étude sur le récit de rêve dans la littérature romanesque des XII et XIII siècles. Paris : Honoré Champion.

Demaules Mireille (dir.) (2016). Expériences oniriques dans la littérature et les arts du Moyen Âge au XVIII siècle. Paris, Honoré Champion.

DIÉNY Jean-Pierre (2001). « Le saint ne rêve pas ». Études chinoises, XX, printemps/ automne.

DiETERLE Bernard et ENGEL Manfred (dir.) (2003). The Dream and the Enlightenment. Paris, Honoré Champion.

DiETERLE Bernard et ENGEL Manfred (dir.) (2018). Theorizing the Dream. Savoirs et théories du rêve. Würzburg, Königshausen \& Neumann. 
DUMORA-MABILLE Florence (2003). « Faire l'histoire du rêve ». In DAUVOIS Nathalie et GROSPERRIN Jean-Philippe (dir.) (2003), Songes et songeurs (XIII-XVIII siècles). Laval, Presses de l'Université de Laval.

DUMORA Florence (2005). L'Euvre nocturne. Songe et représentation au XVII' siècle. Paris, Honoré Champion.

EDELMAN Nicole (1995). Voyantes, guérisseuses et visionnaires en France. 1785-1914. Paris, Albin Michel.

EDELMAN Nicole (2006). Histoire de la voyance et du paranormal. Paris, Seuil.

ERMISSE Gérard (dir.) (1996). « Rêver ». Terrain, 26.

FABRE Daniel (1996). « Rêver. Le mot, la chose, l'histoire ». In ERMISSE Gérard (dir.), Terrain, 26 («Rêver») : 69-82.

FAHD Toufic (1966). La Divination arabe. Études religieuses, sociologiques et folkloriques sur le milieu natif de l'Islam. Strasbourg, Université de Strasbourg.

FREUD Sigmund (1981). « Lettre à Maxime Leroy sur quelques rêves de Descartes »

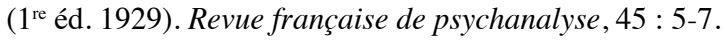

FREUD Sigmund (2010). L'Interprétation du rêve (1 ${ }^{\text {re }}$ éd. 1900), traduit par J.P. LEFEBVRE. Paris, Seuil.

GANTET Claire (2010a). Der Traum in der Frühen Neuzeit. Ansätze zu einer kulturellen Wissenschaftgeschicht. Berlin, De Gruyter.

GANTET Claire (2010b). « Le rêve dans l'Allemagne du XVI ${ }^{\mathrm{e}}$ siècle. Appropriations médicales et recouvrements confessionnels ». Annales. Histoire, sciences sociales, 65(1) : 39-62.

GAUTIER Jean-Luc (dir.) (1988). « Rêver en France au XVII siècle ». Revue des Sciences Humaines, 211.

Gollut Jean-Daniel (1993). Conter les rêves. La narration de l'expérience onirique dans les œuvres de la modernité. Paris, José Corti.

HeRVEY DE SAINT-DENYs Léon (D’) (1867). Les Rêves et les moyens de les diriger. Observations pratiques. Paris, Amyot.

Huntington Rania (2017). «Rêves des morts : cas tirés des Annales de la famille Yu de Dequing », trad. V. DuRAND-DASTÈS. In Fantômes dans l'Extrême-Orient d'hier et d'aujourd'hui, 1. Paris, Presses de l'Inalco.

Kruger Stefen F. (1992). Dreaming in the Middle Ages. Cambridge, Cambridge University Press.

LAHIRE Bernard (2018). L'Interprétation sociologique des rêves. Paris, La Découverte.

LE GoFF Jacques (1977). « Les rêves dans la culture et dans la psychologie collective de l'Occident médiéval ». In Pour un autre Moyen Âge. Temps, travail et culture en Occident : dix-huit essais. Paris, Gallimard : 299-306.

LE GoFf Jacques (1985). «Le christianisme et les rêves (II ${ }^{\mathrm{e}}-\mathrm{VII}{ }^{\mathrm{e}}$ siècles) 》. In L'Imaginaire médiéval. Paris, Gallimard : 265-316.

LORY Pierre (2003). Le Rêve et ses interprétations en Islam. Paris, Albin Michel.

MACARIO Maurice (1857). Du sommeil, des rêves et du somnambulisme dans l'état de santé et de maladie. Lyon/Paris, Périsset Frères.

MARINELLI Lydia et MAYER Andreas (2009). Rêver avec Freud. L'histoire collective de L'interprétation du rêve, traduit par D. TASSEL. Paris, Aubier-Flammarion. 
MAURY Alfred (1860). La Magie et l'astrologie dans l'Antiquité et au Moyen Âge ou étude sur les superstitions païennes qui se sont perpétuées jusqu'à nos jours. Paris, Didier.

MAURY Alfred (1861). Le Sommeil et les rêves. Études psychologiques sur ces phénomènes et les divers états qui s'y rattachent. Paris, Didier.

MAURY Alfred (1862). «Songe (Psychologie)». In Encyclopédie moderne. Dictionnaire abrégé des sciences, des lettres, des arts, de l'industrie, de l'agriculture et du commerce. Paris, Firmin Didot, vol. 25 : 584-592.

MAYER Andreas (2016). «La Traumdeutung, clé des songes du XX $\mathrm{XX}^{\mathrm{e}}$ siècle ? Freud, Artémidore et les avatars de la symbolique onirique ». In CARROY Jacqueline et LANCEL Juliette (dir.), Clés des songes et sciences des rêves. De l'Antiquité à Freud. Paris, Les Belles Lettres : 157-181.

PERRIN Michel (2011). Les Praticiens du rêve. Un exemple de chamanisme. Paris, PUF.

PICK Daniel et RoPER Lyndal (dir.) (2004). Dreams and History. The Interpretation of Dreams from Ancient Greece to Modern Psychoanalysis. Londres/New York, Brunner/Routledge.

PINO Angel (1995). «Abrégé dûment circonstancié de la vie de Marie Jean Léon Le Coq, baron d'Hervey, marquis de Saint-Denys, professeur au Collège de France et membre de l'Institut, sinologue de son état, onironaute à ses heures. Une enquête à l'usage non exclusif des futurs biographes ». In BERGÈRE Marie-Claire et PINO Angel (dir.), Un siècle d'enseignement du chinois à l'École des langues orientales. 1840-1945. Paris, L'Asiathèque : 95-129.

PonTAS Jean (1847). «Songe ». In Dictionnaire de cas de conscience, ou Décisions, par ordre alphabétique, des plus considérables difficultés touchant la morale et la discipline ecclésiastique II. Paris, Migne : 667-668.

PORRET Michel (2001). L'Homme aux pensées nocturnes. Pierre Frémont, libraire et explicateur de rêves à Genève au siècle des Lumières. Genève, Métropolis.

RIPA Yannick (1988). Histoire du rêve. Regards sur l'imaginaire des Français au XIX $X^{e}$ siècle. Paris, Olivier Orban.

SchmitT Jean-Claude (2001). Le Corps, les rites, les rêves, le temps. Essais d'anthropologie médiévale. Paris, Gallimard.

TiмотіN Andrei (2010). Visions, prophéties et pouvoir à Byzance. Étude sur l'hagiographie méso-byzantine ( $I X^{e}-X I^{e} s$.). Paris, Éditions de l'EHESS.

VATAN Florence (2017). « Gustave Flaubert ou l'alambic des rêves ». In CARROY Jacqueline (dir.), Romantisme, 178 (« Rêver au XIX ${ }^{\mathrm{e}}$ siècle ») : 50-61. 